\title{
A Rare Pulmonary Arterio-venous Malformation with Circular Shunting Due to Inferior Phrenic Artery Supply with Pulmonary Venous Drainage: a Case Report
}

\author{
James Whiteley Dobson ${ }^{1,2} \cdot$ Edem Barnor-Ahiaku $^{1,2} \cdot$ Sapna Puppala ${ }^{1,2} \cdot$ Simon John McPherson ${ }^{1,2}$ (D)
}

Accepted: 13 January 2021 / Published online: 28 January 2021

(C) Crown 2021

\begin{abstract}
A rare case of a pulmonary arterio-venous malformation not requiring treatment is reported. An incidental peripheral right lower lobe intrapulmonary pulmonary arterio-venous malformation was identified on computed tomography. On catheter angiography, no pulmonary artery supply was identified. Systemic artery catheter arteriography identified systemic arterial supply from a conjoined inferior phrenic artery with pulmonary venous drainage to the left atrium. Due to this lung abnormality being a systemic arterial circulation to pulmonary venous circulation (left to left or circular) shunt, no treatment was indicated.
\end{abstract}

Keywords Pulmonary arterio-venous malformations (PAVMs) · Computed tomography (CT) · Catheter angiography/ arteriography $\cdot$ Systemic to pulmonary shunt $\cdot$ Left to left shunt $\cdot$ Circular shunt $\cdot$ Left to right shunt $\cdot$ Right to left shunt

\section{Introduction}

Pulmonary arterio-venous malformations (PAVMs) are usually due to an abnormal connection between the pulmonary arteries and pulmonary veins resulting in a right to left shunt. Around $90 \%$ of patients with PAVMs have an associated genetic syndrome, most commonly hereditary haemorrhagic telangiectasia (HHT). Solitary PAVMs are more likely to be sporadic in aetiology. Large or multiple PAVMs may cause hypoxaemia or high-output cardiac failure. These complications do not occur with small solitary PAVMs. Any PAVM may result in haemoptysis. It is now generally accepted that all PAVMs, irrespective of the size of the feeding vessel, require treatment due to the risk of paradoxical embolic events (including stroke and brain abscess) secondary to the right to left

This article is part of the Topical Collection on Imaging

Simon John McPherson

simon.mcpherson@nhs.net

1 Department of Vascular Interventional Radiology, Radiology Department, Jubilee Wing, Leeds General Infirmary, Leeds Teaching Hospitals Trust, Great George Street, Leeds LS1 3EX, UK

2 Department of Interventional Radiology, Jubilee Wing, Leeds General Infirmary, Leeds Teaching Hospitals, University of Leeds, Great George St, Leeds LS2 9JT, UK shunt [1] [2]. Endovascular embolisation is the first line treatment modality.

\section{Case Presentation}

A 60-year-old male smoker presented with progressive SOB and a persistent productive cough. His chest radiograph was unremarkable and he subsequently had a chest CT which identified an incidental asymptomatic PAVM peripherally in the right lung lower lobe at the right costophrenic recess (Fig. 1). There was no history of trauma, surgery, endocarditis or severe pulmonary infection (tuberculosis, fungal or bacterial). The patient was referred to the interventional radiology (IR) clinic for consideration of embolisation. CT scan reviewed in clinic by the consulting interventional radiologist was considered to show a connection between a right lower lobe pulmonary artery and vein. Informed consent for embolisation was obtained in the IR clinic.

Pulmonary arteriography was performed via a 5Fr right common femoral vein sheath under local anaesthesia with underwater catheter and guidewire exchanges (Fig. 2a, b). Extensive right-sided pulmonary catheter angiography was unable to identify a PAVM (Fig. 3a). Pulmonary artery pressures were normal. This prompted on table review of an earlier CT scan from 2014 which had better systemic arterial opacification; the patient had undergone this scan for 
Fig. 1 a, b Sequential slices of coronal CT showing vascular sac (black arrows) and draining vein (white arrows) in the right lower lobe
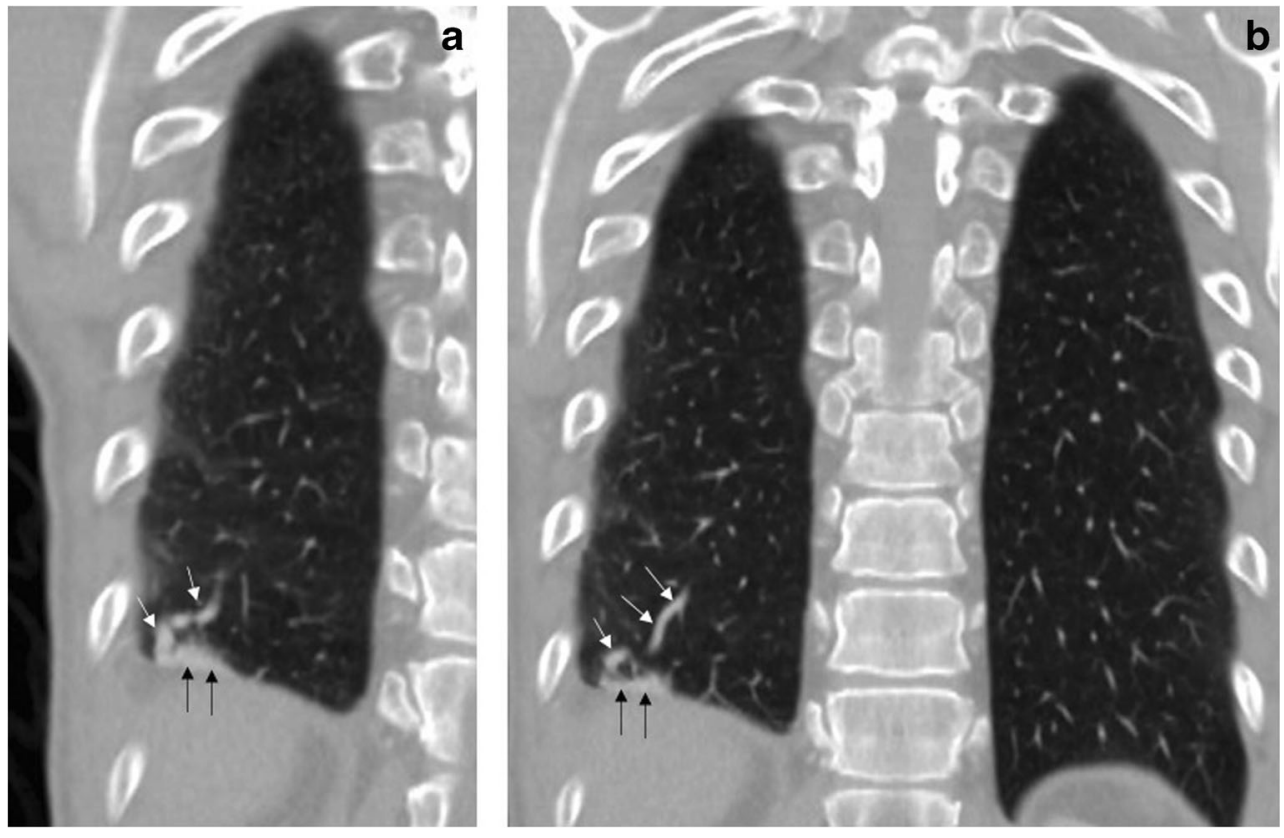

unrelated indications of "weight loss? cause". This identified an abnormal tortuous right inferior phrenic artery directed towards the PAVM (Fig. 3b). Diagnostic systemic arteriography was then performed via a $5 \mathrm{Fr}$ right common femoral artery sheath.

The inferior phrenic arteries had a conjoined origin with a hypertrophied right inferior phrenic artery supplying the right basal lung juxta-diaphragmatic PAVM with pulmonary venous drainage to the left atrium (Fig. 4a-d). The PAVM produced a left to left or circular shunt. Due to a proximal stenosis in the right phrenic artery and the conjoined inferior phrenic artery origin, significant collateral supply to the PAVM was from a tortuous branch of the left phrenic artery. This led to hypertrophy of the left phrenic artery as well as the right. Branches of the dorsal pancreatic artery also provided supply (Fig. 4a). Following discussion with the patient, a conservative approach was taken given that the PAVM was stable/ longstanding, had complex anatomy, had no complications (no haemoptysis or cardiac failure), and carried an extremely low risk of systemic emboli. The patient and his general practitioner are aware of the findings and the need to re-refer to the IR clinic should haemoptysis develop. He remained asymptomatic in the subsequent 18 months.

\section{Conclusions}

Cardiovascular shunts are divided into intra-cardiac and extracardiac. The commonest extra-cardiac shunts are right to left shunts which include PAVMS, hepatopulmonary syndrome, congenital cardiac disease and post-traumatic fistulas. Left to right extra-cardiac shunts are rarer (e.g. total or partial anomalous pulmonary venous drainage). Even rarer are shunts which do not cross a systemic capillary bed (a left to left shunt) or pulmonary capillary bed (a right to right shunt). These are also known as circular shunts. Anomalous systemic
Fig. 2 Photos showing the underwater catheter exchanges. a A continuous flush system is attached to the long-sheath (black arrow). $\mathbf{b}$ To avoid aspiration of air and paradoxical air embolisation, particularly to the cerebral circulation, all catheter and wire exchanges are performed underwater
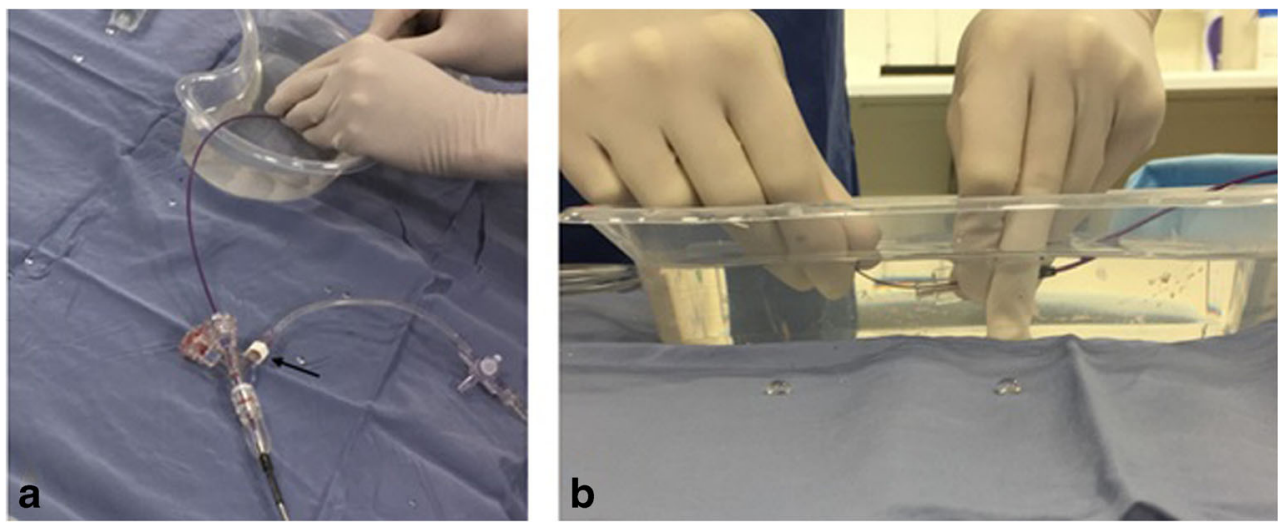
Fig. 3 a Multiple attempts of selective pulmonary artery angiography were performed, none demonstrating an AVM. b Re-review of CT images showing a communication between the right inferior phrenic artery (black arrows) and the prominent pulmonary vasculature (white arrow)
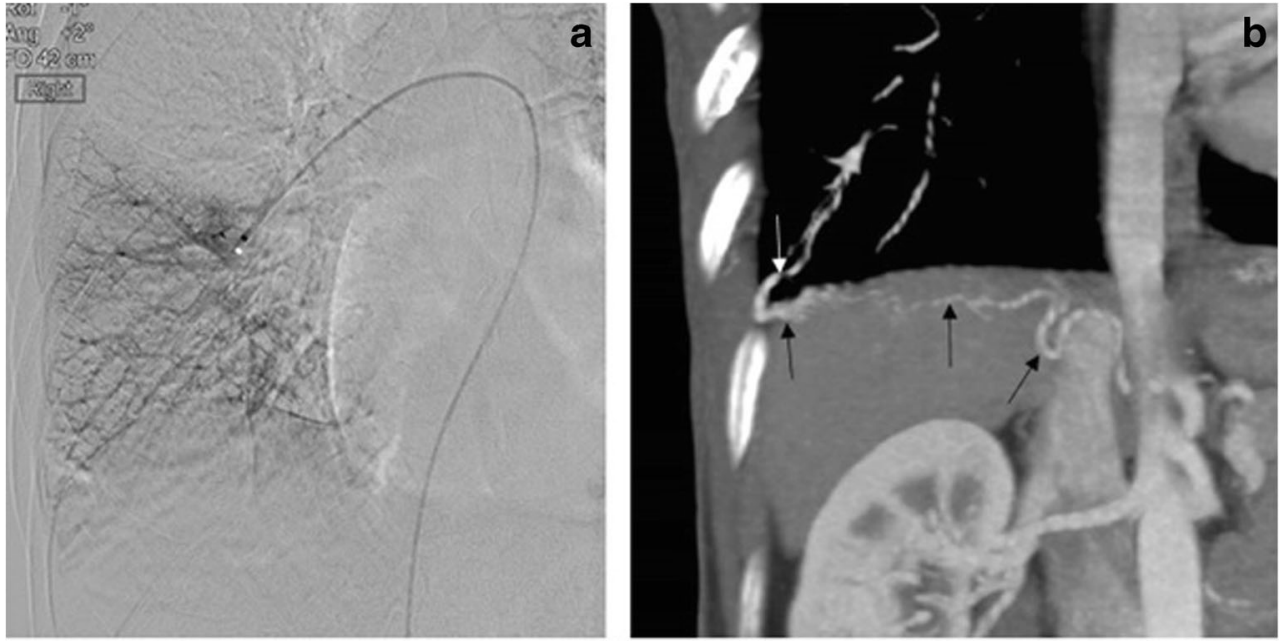

artery to pulmonary venous malformations/fistulae are extremely rare [3].

Paradoxical emboli occur in pulmonary artery to pulmonary vein PAVMs due to venous thrombo-emboli shunting through the PAVM and bypassing the filter effect of the pulmonary capillary bed. Usually systemic circulation thromboembolism arises from the left atrium, left ventricle, aorta or large vessels. A PAVM with a circular shunt might minimally increase the risk of stroke by re-circulating emboli. Thrombus formation in the PAVM sac is rare but could potentially result in a systemic embolus [4]. No documented reports of this occurrence were identified in the literature. The higher flow systemic supply should make thrombus formation less likely in our case than in the higher prevalence right to left PAVMs.
Fig. 4 Angiography showing (a) access into the inferior phrenic arteries (there is a stenosis of the right artery (solid arrow) with collateral arcade from the left artery (dashed arrow)); (b) proximal filling of the right inferior phrenic artery; (c) contrast blush of the AVM nidus (white arrow); (d) draining of the $A V M$ via the inferior right pulmonary vein (white dashed arrow) into the left atrium (not shown)
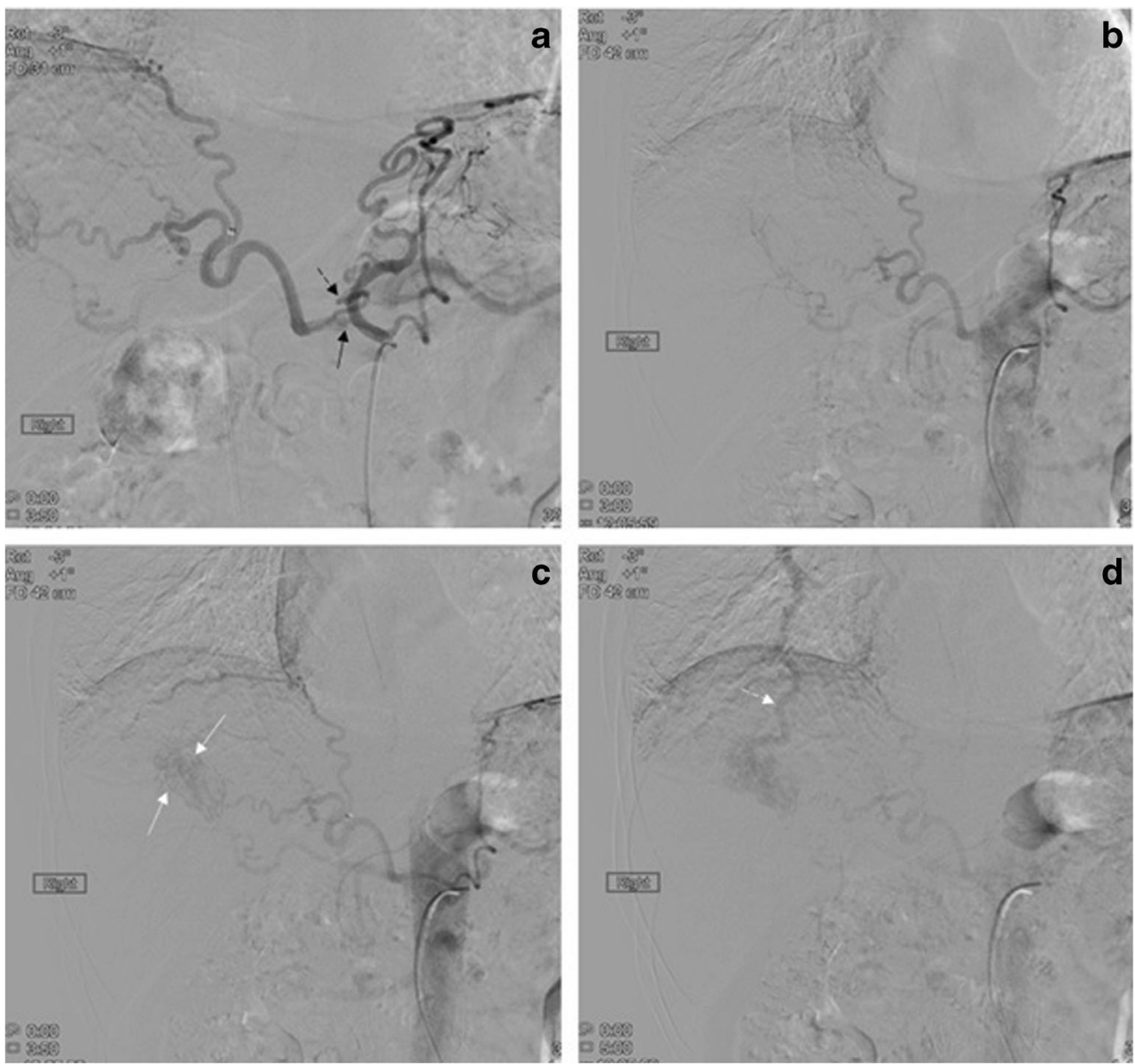
Rarely HHT PAVMs may have dual pulmonary and systemic arterial supply [5].

In this case, the underlying lung was normal excluding hypogenetic lung syndrome and intrapulmonary sequestration which may result in circular shunts [6]. It is recognised that systemic arteries may supply normal lung with normal pulmonary venous drainage, most commonly at the left lung base [7]. This is not the scenario in this case as there is an abnormal vascular sac (arrowed in Fig. 1).

Congenital systemic artery to pulmonary vein connections, the presumed aetiology in this case report, are rare. Jariwala and colleagues identified 21 cases in 16 papers when they reviewed the literature in 2014 [8]. The majority of these PAVMs arose from unnamed vessels arising from the aorta with one case coming from the left lateral thoracic artery branch of the left subclavian artery. Thirteen cases were treated due to highoutput cardiac failure. Four cases were treated with no reason given, one patient died, one paper was not found and the remaining two were not treated. Historically, treatment was with open surgery, but more recent cases were controlled endovascularly. Treatment was refused in two cases identified by Jariwala's review who were asymptomatic, one a 5-year-old and a 55-year-old. Neither had long-term follow-up.

A similar incidental PAVM in a 52-year-old with inferior phrenic and internal mammary arterial supply but connection to the pulmonary artery rather than vein was reported by Geyik et al. in 2006 [9]. The authors embolised this asymptomatic PAVM by occluding the draining pulmonary artery branch despite the anatomical connections not intuitively representing a risk of paradoxical embolisation. The authors considered that embolisation was indicated due to a future risk of haemoptysis and pulmonary hypertension despite the history pointing to a congenital lesion which had not caused symptoms in 52 years. The patient in this report is not at risk of pulmonary arterial hypertension as the systemic connection was to a right lower lobe pulmonary vein. Embolisation has risks of pulmonary infarction, infection and damage to accessed vessels. The "first do no harm principle" indicates that a treatment should only be offered where the chances of benefit exceed the risk of harm. The absence of any literature or significant anatomical risk of paradoxical embolism, to indicate that intervention is justified, led us to adopting a conservative observant approach with the patient's agreement. The patient is under review such that we can report if this strategy was the wrong choice.

\section{Code Availability Not applicable.}

Author's contribution Dr James Dobson was in charge of literature review and wrote up the paper, Edem Barnor-Ahiaku was in charge of literature review and wrote up the paper, Dr Sapna Puppala supervised the case write up and Dr Simon John McPherson performed the angiography and supervised the case write up.

\section{Compliance with ethical standards}

Conflict of Interest The authors declare no competing interests

Ethics Approval and Consent to Participate Not applicable.

Consent for Publication Written consent was obtained from the patient

Open Access This article is licensed under a Creative Commons Attribution 4.0 International License, which permits use, sharing, adaptation, distribution and reproduction in any medium or format, as long as you give appropriate credit to the original author(s) and the source, provide a link to the Creative Commons licence, and indicate if changes were made. The images or other third party material in this article are included in the article's Creative Commons licence, unless indicated otherwise in a credit line to the material. If material is not included in the article's Creative Commons licence and your intended use is not permitted by statutory regulation or exceeds the permitted use, you will need to obtain permission directly from the copyright holder. To view a copy of this licence, visit http://creativecommons.org/licenses/by/4.0/.

\section{References}

1. Saboo SS, Chamarthy M, Bhalla S, Park H, Sutphin P, Kay F, et al. Pulmonary arteriovenous malformations: diagnosis. Cardiovasc Diagn Ther. 2018;8(3):325-37.

2. Müller-Hülsbeck S, Marques L, Maleux G, Osuga K, Pelage J, Wohlgemuth WA, et al. CIRSE standards of practice on diagnosis and treatment of pulmonary arteriovenous malformations. Cardiovasc Intervent Radiol. 2020;43:353-61.

3. Currarino G, Willies K. Congenital fistula between an aberrant systemic artery and a pulmonary vein with sequestration: a report of three cases. J Pediatr. 1975;87(4):554-7.

4. Lacombe P, Lacout A, Marcy P, Binsse S, Sellier J, Bensalah M, et al. Diagnosis and treatment of pulmonary arteriovenous malformations in hereditary hemorrhagic telangiectasia: an overview. Diagn Interv Imaging. 2013;94(9):835-48.

5. Laffey KJ, Thomashow B, Jaretzki A III, Martin EC. Systemic supply to a pulmonary arteriovenous malformation: a relative contraindication to surgery. Am J Roentgenol. 1985;145(4):720-2.

6. Martinez-Jimenez S, Heyneman LE, Page McAdams H, Jasinowodolinksik D, Rossi SE, Restrepo CS, et al. Nonsurgical extracardiac vascular shunts in the thorax: clinical and imaging characteristics. Radiographics. 2010;30(5):e41.

7. Ashizawa K, Ishida Y, Matsunaga N, Otsuji H, Sakamoto I, Hayashi $\mathrm{K}$. Anomalous systemic arterial supply to normal basal segments of left lower lobe: characteristic imaging findings. J Comput Assist Tomogr. 2001;25:764-9.

8. Jariwala P, Ramesh G, Chandra K. Congenital anomalous/aberrant systemic artery to pulmonary venous fistula: closure with vascular plugs \& coil embolization. Indian Heart J. 2014;66(1):95-103.

9. Geyik S, Yavuz K, Keller F. Unusual systemic artery to pulmonary artery malformation without evidence of systemic disease, trauma or surgery. Cardiovasc Intervent Radiol. 2006;29(5):897-901.

Publisher's Note Springer Nature remains neutral with regard to jurisdictional claims in published maps and institutional affiliations.

Data Availability Not applicable. 